\title{
SOME SMOOTH COMPACTLY SUPPORTED TIGHT WAVELET FRAMES WITH VANISHING MOMENTS
}

\author{
A. SAN ANTOLÍN AND R. A. ZALIK
}

\begin{abstract}
Let $A \in \mathbb{R}^{d \times d}, d \geq 1$ be a dilation matrix with integer entries and $|\operatorname{det} A|=2$. We construct several families of compactly supported Parseval framelets associated to $A$ having any desired number of vanishing moments. The first family has a single generator and its construction is based on refinable functions associated to Daubechies low pass filters and a theorem of Bownik. For the construction of the second family we adapt methods employed by Chui and He and Petukhov for dyadic dilations to any dilation matrix $A$. The third family of Parseval framelets has the additional property that we can find members of that family having any desired degree of regularity. The number of generators is $2^{d}+d$ and its construction involves some compactly supported refinable functions, the Oblique Extension Principle and a slight generalization of a theorem of Lai and Stöckler. For the particular case $d=2$ and based on the previous construction, we present two families of compactly supported Parseval framelets with any desired number of vanishing moments and degree of regularity. None of these framelet families have been obtained by means of tensor products of lower-dimensional functions. One of the families has only two generators, whereas the other family has only three generators. Some of the generators associated with these constructions are even and therefore symmetric. All have even absolute values.
\end{abstract}

\section{INTRODUCTION}

The purpose of this paper is to construct symmetric smooth compactly supported tight wavelet frames in $L^{2}\left(\mathbb{R}^{d}\right)$ having any desired number of vanishing moments associated to a general dilation matrix and such that the number of generators does not depend on the number of vanishing moments and the degree of regularity. None of these framelet families have been obtained by means of tensor products of lower-dimensional functions.

The construction of multivariate compactly supported wavelet frames is an interesting problem, both from the theoretical and the applied points of view. Compactly supported wavelets and wavelets frames constructed by univariate tensor product of wavelets (i.e., separable wavelets) have been used widely. However, Belogay and Wang ([2]) remark that although separable wavelet bases are easy to construct and simple to study, they nevertheless have a number of drawbacks. Referring specifically to image processing in $\mathbb{R}^{2}$ they point out that such wavelets have very little design freedom, and that separability imposes an unnecessary product structure on the plane, which is artificial for natural images. This can create unpleasant

Key words and phrases. Dilation matrix, Fourier transform, refinable function, tight framelet, Unitary Extension Principle.

The first author was partially supported by MEC/MICINN grant \#MTM2011-27998 (Spain).

2010 Mathematics Subject Classification: 42C40. 
artifacts that become obvious at high image compression ratios. Velisavljević et al. ([49]) note that one-dimensional discontinuities in images (edges and contours) that are very important elements in visual perception, intersect too many wavelet basis functions and lead to a nonsparse representation, i.e, a large number of nonzero transform coefficients. To avoid this, one usually uses wavelet frames associated to a general dilation or nonseparable wavelets. Numerous papers have been written on the construction of nonseparable wavelets, some influenced in part by Kovačević and Vetterli's seminal paper [29]. In view of these remarks, and bearing in mind the redundancy inherent in representation by frames, it is clear that the study of wavelet frames associated to a general dilation may be useful in practical applications.

The construction of tight wavelet frames has been discussed in an extensive and rich literature. A relationship between vanishing moments of a framelet and good approximation properties was shown, for instance, by Daubechies, Han, Ron and Shen [13]. In dimension one and with dyadic dilation, Daubechies [11] constructed compactly supported orthonormal wavelets with any number of vanishing moments and any degree of regularity. For the multivariate case with a general dilation matrix, it is still not known if there exist compactly supported smooth wavelets with an arbitrary number of vanishing moments. Even for tight wavelet frames, the problem of constructing compactly supported smooth tight wavelet frames having vanishing moments and a fixed number of generators is open.

Han [22] proved the existence of tight wavelet frames associated to any dilation matrix on $\mathbb{R}^{d}$, such that their generators are compactly supported, with degree of smoothness and vanishing moments of order as large as desired, and their number bounded by a constant depending on the dimension and the determinant of the dilation matrix, whereas Gröchenig and Ron [19] and Ron and Shen [38], [39] found constructions of compactly supported tight framelets with any desired degree of smoothness.

Associated to dyadic dilations, Chui, He and Stöckler [7] introduced the notion of vanishing moment recovery function and applied it to the construction of compactly supported tight wavelet frames with two generators to achieve the maximum order of vanishing moments as allowed by an associated refinable function. Among other significant results, Han and Mo [27] show how from a compactly supported real-valued refinable function with stable shifts one can construct a tight wavelet frame set with three generators having the highest possible order of vanishing moments. Related to this paper, there are several constructions by Chui and He [6], Selesnick [44], Ayache [1], Petukhov [34], [35], Dong and Shen [14], Han [26], [25] and Salvatori and Soardi [40]. Compactly supported wavelets with dilation factor 3 are constructed in Chui and Lian [9]. For dilation factor 4 see Han [21]. The paper by Chui, He, Stöckler and Sun [8] deals with the construction of compactly supported tight wavelet frames with many vanishing moments associated to integral factor dilations. In the same context, Petukhov [36] develops an algorithm for constructing tight wavelet frames from a given refinable function with minimal number of generators and supports of minimal size. With a more general dilation matrix, Skopina [46] (see also [47], [45]) describes an algorithm to construct compactly supported wavelet frames with vanishing moments. In Krivoshein [30], wavelet frame systems providing any desired approximation order are constructed for any matrix dilation. 
With a general dilation matrix, there are constructions of wavelet bi-frames. For instance, see Ehler [15] and Ehler and Han [16].

In [41], San Antolín and Zalik developed a method to generate wavelet frames using the Oblique Extension Principle and a slight generalization of a theorem of Lai and Stöckler [32]. This method was used in [42] to construct, for a $2 \times 2$ expansive dilation matrix with integer entries and determinant \pm 2 , families of compactly supported tight framelets with three generators and with any desired degree of smoothness. The same method was used in [43] to construct compactly supported tight framelets having the following additional properties: both the framelets and the refinable functions that generate them can be made as smooth as desired; moreover, these refinable functions are nonseparable, in the sense that they cannot be expressed as the product of two functions defined on lower dimensions.

In this paper we will use the same method to construct smooth compactly supported Parseval framelets and refinable functions with good approximation properties in $L^{2}\left(\mathbb{R}^{d}\right), d \geq 1$, associated to a $d \times d$ expansive dilation matrix $A$ with integer entries and $|\operatorname{det} A|=2$. In Section 3 we construct a family of compactly supported Parseval framelets with one or two generators and any desired number of vanishing moments. In Section 4 we construct families of compactly supported Parseval framelets with generators that have any given number of vanishing moments and any desired degree of regularity. For this family, the number of generators is $2^{d}+d$, and therefore does not depend on the regularity or the number of vanishing moments. For $d=2$ this allows us to construct Parseval framelets with six generators.

However, in the fifth and last section we present families of Parseval framelets with only two or three generators and $d=2$. Apart from other implications, this improves our results in [42] by adding the additional property of having any desired number of vanishing moments.

\section{Notation, Definitions, and Underlying Assumptions}

We now introduce our notation, definitions, and underlying assumptions.

The sets of strictly positive integers, integers, rational numbers and real numbers will be denoted by $\mathbb{N}, \mathbb{Z}, \mathbb{Q}$ and $\mathbb{R}$ respectively. We will write $\mathbf{t}=\left(t_{1}, \ldots, t_{d}\right)^{T} \in \mathbb{R}^{d}$ and $\mathbf{x}=\left(x_{1}, \ldots, x_{d}\right)^{T} \in \mathbb{R}^{d}$. Unless otherwise stated, we will assume that $n, m \in \mathbb{N}$.

Given a matrix $A$, its transpose will be denoted by $A^{T}$, and the conjugate of its transpose by $A^{*}$.

We say that $A \in \mathbb{R}^{d \times d}$ is a dilation matrix preserving the lattice $\mathbb{Z}^{d}$ if all its eigenvalues have modulus greater than 1 and $A \mathbb{Z}^{d} \subset \mathbb{Z}^{d}$. The set of all $d \times d$ dilation matrices preserving the lattice $\mathbb{Z}^{d}$ will be denoted by $\mathbf{E}_{d}(\mathbb{Z})$. Note that if $A \in \mathbf{E}_{d}(\mathbb{Z})$ then $|\operatorname{det} A|$ is an integer greater than 1 , and the quotient groups $\mathbb{Z}^{d} / A \mathbb{Z}^{d}$ and $A^{-1} \mathbb{Z}^{d} / \mathbb{Z}^{d}$ are well defined. From [18, Lemma 2$]$ we know that $\mathbb{Z}^{d} / A \mathbb{Z}^{d}$ has exactly $|\operatorname{det} A|$ cosets, which readily implies that also $A^{-1} \mathbb{Z}^{d} / \mathbb{Z}^{d}$ has exactly $|\operatorname{det} A| \operatorname{cosets}$. With the exception of Theorems B, D, and E, or unless otherwise stated, will assume throughout that $A \in \mathbf{E}_{d}(\mathbb{Z})$ is such that $|\operatorname{det} A|=2$, By $\boldsymbol{\Gamma}_{A}=\left\{\mathbf{r}_{0}(A), \mathbf{r}_{1}(A)\right\}$ we will denote a full collection of representatives of the cosets of $\left(A^{T}\right)^{-1} \mathbb{Z}^{d} / \mathbb{Z}^{d}$ such that $\mathbf{r}_{0}(A)=(0, \ldots, 0)^{T}$ and $\mathbf{r}_{1}(A)=\left(r_{1}^{(1)}, \ldots, r_{1}^{(d)}\right)^{T} \in\{0,1 / 2\}^{d}$. The existence of $\boldsymbol{\Gamma}_{A}$ is proved in Lemma 1 below. The letter $a$ as a subscript will always stand for an index in $\{1, \ldots, d\}$ such that $r_{1}^{(a)}=1 / 2$. 
4 SMOOTH COMPACTLY SUPPORTED TIGHT FRAMELETS WITH VANISHING MOMENTS

Let $\widehat{f}$ denote the Fourier transform of the function $f$. Thus, if $f \in L^{1}\left(\mathbb{R}^{d}\right)$ and $\mathbf{x}, \mathbf{t} \in \mathbb{R}^{d}$, then

$$
\widehat{f}(\mathbf{t}):=\int_{\mathbb{R}^{d}} f(\mathbf{x}) e^{-2 \pi i \mathbf{x} \cdot \mathbf{t}} d \mathbf{x}
$$

where $\mathbf{x} \cdot \mathbf{t}$ denotes the dot product of vectors $\mathbf{x}$ and $\mathbf{t}$. The Fourier transform is extended to $L^{2}\left(\mathbb{R}^{d}\right)$ in the usual way.

A sequence $\left\{\phi_{n}\right\}_{n=1}^{\infty}$ of elements in a separable Hilbert space $\mathbb{H}$ is a frame for $\mathbb{H}$ if there exist constants $C_{1}, C_{2}>0$ such that

$$
C_{1}\|h\|^{2} \leq \sum_{n=1}^{\infty}\left|\left\langle h, \phi_{n}\right\rangle\right|^{2} \leq C_{2}\|h\|^{2}, \quad \forall h \in \mathbb{H},
$$

where $\langle\cdot, \cdot\rangle$ denotes the inner product on $\mathbb{H}$. The constants $C_{1}$ and $C_{2}$ are called frame bounds. The definition implies that a frame is a complete sequence of elements of $\mathbb{H}$. A frame $\left\{\phi_{n}\right\}_{n=1}^{\infty}$ is tight if we may choose $C_{1}=C_{2}$.

Let $A$ be any dilation matrix in $\mathbf{E}_{d}(\mathbb{Z})$. A set of functions $\Psi=\left\{\psi_{1}, \ldots, \psi_{N}\right\} \subset$ $L^{2}\left(\mathbb{R}^{d}\right)$ is called a wavelet frame or framelet with dilation $A$, if the system

$$
\left\{\psi_{\ell, j, \mathbf{k}}(\mathbf{x}) ; j \in \mathbb{Z}, \mathbf{k} \in \mathbb{Z}^{d}, 1 \leq \ell \leq N\right\},
$$

where $\psi_{\ell, j, \mathbf{k}}(\mathbf{x}):=|\operatorname{det} A|^{j / 2} \psi_{\ell}\left(A^{j} \mathbf{x}+\mathbf{k}\right)$, is a frame for $L^{2}\left(\mathbb{R}^{d}\right)$. If this system is a tight frame for $L^{2}\left(\mathbb{R}^{d}\right)$ then $\Psi$ is called a tight wavelet frame or tight framelet. If the functions $\psi_{\ell}, \ell=1, \ldots N$ are linearly independent they are called the generators of the framelet. If the frame constant is equal to 1 it will be called a Parseval framelet in $L^{2}\left(\mathbb{R}^{d}\right)$. Thus we have:

$$
\sum_{\ell=1}^{N} \sum_{j \in \mathbb{Z}} \sum_{\mathbf{k} \in \mathbb{Z}^{d}}\left|\left\langle f, \psi_{\ell, j, \mathbf{k}}\right\rangle\right|^{2}=\|f\|^{2} \quad \forall f \in L^{2}\left(\mathbb{R}^{d}\right) .
$$

A wavelet frame $\Psi=\left\{\psi_{1}, \ldots, \psi_{N}\right\} \subset L^{2}\left(\mathbb{R}^{d}\right)$ has vanishing moments of order $m \in\{0,1, \cdots\}$, if $\widehat{\psi}_{\ell}, \ell=1, \cdots, N$ has a zero of order $m$ at the origin.

\section{Compactly supported tight Framelets With VANishing MOMEnts}

In this section we construct Parseval framelets associated to $A$ with vanishing moments of order $n$, and having one or two generators. In our construction we will use the following trigonometric polynomials in $\mathbb{R}$. For $n=1,2,3, \cdots$, let

$$
g_{n}(t):=1-c_{n} \int_{0}^{t}(\sin 2 \pi \xi)^{2 n+1} d \xi,
$$

where $c_{n}=\left(\int_{0}^{1 / 2}(\sin 2 \pi \xi)^{2 n+1} d \xi\right)^{-1}$. These polynomials satisfy the following identity:

$$
g_{n}(t)+g_{n}(t+1 / 2)=1 .
$$

For convenience, we now list additional properties of the functions $g_{n}$ :

Lemma A. ([50, Lemma 4.8]) For $n=1,2, \cdots$, the trigonometric polynomials $g_{n}$ satisfy the following:

(a) $g_{n}(0)=1$;

(b) $g_{n}(t)>0$ if $t \notin 1 / 2+\mathbb{Z}$;

(c) $g_{n}(t)=0$ if $t \in 1 / 2+\mathbb{Z}$; 
(d) $g_{n}$ is $\mathbb{Z}$-periodic and even.

From a lemma of Riesz ([12, Lemma 6.1.3] or [33, Lemma 10, p. 102]) we know that there exist trigonometric polynomials

$$
q_{n}(t):=\sum_{k=0}^{2 n+1} \alpha_{k}^{(n)} e^{2 \pi i k t}, \quad \alpha_{k}^{(n)} \in \mathbb{C},
$$

such that $\left|q_{n}(t)\right|^{2}=g_{n}(t)$ and $q_{n}(0)=1$. The coefficients of these polynomials may be obtained by spectral factorization ([17]) and may be assumed to be real-valued. From (2) we see that

$$
\left|q_{n}(t)\right|^{2}+\left|q_{n}(t+1 / 2)\right|^{2}=1 .
$$

The following lemma confirms the existence of the collections of representatives $\boldsymbol{\Gamma}_{A}$ of the cosets of $\left(A^{T}\right)^{-1} \mathbb{Z}^{d} / \mathbb{Z}^{d}$ that were defined in the previous section.

Lemma 1. There exists a full collection of representatives

$$
\boldsymbol{\Gamma}_{A}=\left\{\mathbf{r}_{0}(A)=(0, \ldots, 0)^{T}, \mathbf{r}_{1}(A)=\left(r_{1}^{(1)}, \ldots, r_{1}^{(d)}\right)^{T}\right\}
$$

of the cosets of $\left(A^{T}\right)^{-1} \mathbb{Z}^{d} / \mathbb{Z}^{d}$, such that $\boldsymbol{\Gamma}_{A}$ is a proper subset of $\{0,1 / 2\}^{d}$.

Proof. Since the entries of $A^{T}$ are integers, it follows that the entries of $\left(A^{T}\right)^{-1}$ are rational numbers. Thus

$$
\mathbf{r}_{1}:=\left(r_{1}^{(1)}, \ldots, r_{1}^{(d)}\right)^{T} \in \mathbb{Q}^{d} .
$$

Moreover $2 \mathbf{r}_{1} \in \mathbb{Z}^{d}$ or, equivalently, $2 r_{1}^{(\ell)} \in \mathbb{Z}, \ell=1, \ldots, d$, because $\left(A^{T}\right)^{-1} \mathbb{Z}^{d} / \mathbb{Z}^{d}$ is an additive group of order 2 .

Thus $r_{1}^{(\ell)}=(1 / 2) w_{1}^{(\ell)}+k_{1}^{(\ell)}$ where $w_{1}^{(\ell)} \in\{0,1\}$ and $k_{1}^{(\ell)} \in \mathbb{Z}$, and we conclude that $\Gamma$ is a subset of a full collection of representatives of the cosets of $2^{-1} \mathbb{Z}^{d} / \mathbb{Z}^{d}$. Since $2^{-1} \mathbb{Z}^{d} / \mathbb{Z}^{d}$ is an additive group with cardinality $2^{d}$, the assertion follows.

3.1. Tight framelets with one generator. Let us construct tight wavelet frames with one generator and vanishing moments. Our construction is based on refinable functions associated to Daubechies low pass filters and a theorem of Bownik.

We have the following

Proposition 1. Let $n \in \mathbb{N}$ and let

$$
P_{n}(\mathbf{t})=q_{n}\left(t_{a}\right),
$$

where $q_{n}$ is defined by (3). Then the infinite product

$$
\prod_{j=1}^{\infty} P_{n}\left(\left(A^{T}\right)^{-j} \mathbf{t}\right)
$$

converges to a nonzero continuous function $\widehat{\phi}_{n}$ in $L^{2}\left(\mathbb{R}^{d}\right)$ such that $\left\|\widehat{\phi}_{n}\right\|_{L^{2}\left(\mathbb{R}^{d}\right)} \leq 1$, $\widehat{\phi}_{n}(\mathbf{0})=1$, and satisfies the refinement equation

$$
\widehat{\phi}_{n}\left(A^{T} \mathbf{t}\right)=P_{n}(\mathbf{t}) \widehat{\phi}_{n}(\mathbf{t}), \quad \mathbf{t} \in \mathbb{R}^{d} .
$$

Moreover, the function $\phi_{n} \in L^{2}\left(\mathbb{R}^{d}\right)$ whose Fourier transform is $\widehat{\phi}_{n}$ is nonzero and compactly supported, and the functions $\left|\widehat{\phi}_{n}(\mathbf{t})\right|,\left|P_{n}(\mathbf{t})\right|$, and $\left|P_{n}\left(\mathbf{t}+r_{1}(A)\right)\right|$ are even. 
6 SMOOTH COMPACTLY SUPPORTED TIGHT FRAMELETS WITH VANISHING MOMENTS

Proof. Since $\left|q_{n}(t)\right|^{2}=g_{n}(t)$ and $g_{n}(t)$ is even, we readily conclude that $\left|P_{n}(\mathbf{t})\right|$ is even, which in turn readily implies that $\left|\widehat{\phi}_{n}(\mathbf{t})\right|$ is even. By periodicity,

$$
\left|P_{n}\left(-\mathbf{t}+r_{1}(A)\right)\right|=\left|P_{n}\left(\left(-\mathbf{t}-r_{1}(A)\right)+2 r_{1}(A)\right)\right|=\left|P_{n}\left(\mathbf{t}+r_{1}(A)\right)\right| .
$$

Since $P_{n}$ is a trigonometric polynomial on $\mathbb{R}^{d}$ such that $P_{n}(\mathbf{0})=1$ and

$$
\left|P_{n}(\mathbf{t})\right|^{2}+\left|P_{n}\left(\mathbf{t}+\mathbf{r}_{1}\right)\right|^{2}=g_{n}\left(t_{a}\right)+g_{n}\left(t_{a}+1 / 2\right)=1,
$$

the infinite product $\prod_{j=1}^{\infty} P_{n}\left(\left(A^{T}\right)^{-j} \mathbf{t}\right)$ converges pointwise. Thus the assertions follow by [3, Lemma 4 and Theorem 1], except for the compactness of the support of $\widehat{\phi}_{n}$, which is established replicating an argument of Wojtaszczyk [50, p. 79].

Proofs of parts of Proposition 1 for the dyadic case and a single variable may be found in e.g. [4, Corollary 5.1], [12] or [28].

We have:

Theorem 1. Let $\psi_{n} \in L^{2}\left(\mathbb{R}^{d}\right)$ be defined by

$$
\widehat{\psi}_{n}\left(A^{T} \mathbf{t}\right):=e^{2 i \pi t_{a}} \overline{P_{n}\left(\mathbf{t}+\mathbf{r}_{1}(A)\right)} \widehat{\phi}(\mathbf{t}) .
$$

Then $\psi_{n}$ is a compactly supported Parseval framelet in $L^{2}\left(\mathbb{R}^{d}\right)$ with vanishing moments of order $n$, and both the absolute values of $\widehat{\psi}_{n}(\mathbf{t})$ and the generator function $\phi(\mathbf{t})$ are even.

Proof. Let $\mathbf{r}_{1}:=\mathbf{r}_{1}(A)$. A straightforward computation shows that

$$
P_{n}(\mathbf{t}) e^{-2 i \pi t_{a}} P_{n}\left(\mathbf{t}+\mathbf{r}_{1}\right)+P_{n}\left(\mathbf{t}+\mathbf{r}_{1}\right) e^{-2 i \pi\left(t_{a}+1 / 2\right)} P_{n}\left(\mathbf{t}+\mathbf{r}_{1}+\mathbf{r}_{1}\right)=0 .
$$

If $\ell_{0}:=(0, \ldots, 0)$ and $\ell_{1}:=A^{T} \mathbf{r}_{1}$, we readily see that $\left\{\ell_{0}, \ell_{1}\right\}$ is a full collection of representatives of the cosets of $\mathbb{Z}^{d} / A^{T} \mathbb{Z}^{d}$. If $m_{0}(\mathbf{t}):=P_{n}(\mathbf{t})$ and $m_{1}(\mathbf{t}):=$ $e^{2 i \pi t_{a}} \overline{P_{n}\left(\mathbf{t}+\mathbf{r}_{1}\right)}$, then (6) implies that

$$
\left|m_{0}(\mathbf{t})\right|^{2}+\left|m_{1}(\mathbf{t})\right|^{2}=1
$$

(8) implies that

$$
m_{0}(\mathbf{t}) \overline{m_{1}(\mathbf{t})}+m_{0}\left(\mathbf{t}+\left(A^{T}\right)^{-1} \ell_{1}\right) \overline{m_{1}\left(\mathbf{t}+\left(A^{T}\right)^{-1} \ell_{1}\right)}=0,
$$

and applying [3, Lemma 5 and Theorem 4] we conclude that $\psi_{n}$ is a Parseval framelet.

Since $\phi_{n}$ has compact support and the function $e^{2 i \pi t_{a}} \overline{P_{n}\left(\mathbf{t}+\mathbf{r}_{1}\right)}$ is a trigonometric polynomial, it follows that the function $\psi_{n}$ is compactly supported. The function $\left|\widehat{\psi}_{n}(\mathbf{t})\right|$ is even because $\left|\widehat{\phi}_{n}(\mathbf{t})\right|$ and $\left|P_{n}\left(\mathbf{t}+r_{1}(A)\right)\right|$ are even.

We now verify that $\psi_{n}$ has vanishing moments of order $n$. Since the absolute value of the eigenvalues of $A^{T}$ is greater than one, there exists $C>0$ such that $\left\|A^{T} \mathbf{t}\right\| \geq C\|\mathbf{t}\|$. Therefore, since $0 \leq|\widehat{\phi}(\mathbf{t})| \leq 1$, using (6) we have:

$$
\begin{aligned}
\lim _{\mathbf{t} \rightarrow \mathbf{0}} \frac{\left|\widehat{\psi}_{n}\left(A^{T} \mathbf{t}\right)\right|^{2}}{\left\|A^{T} \mathbf{t}\right\|^{2 n}} & \leq \lim _{\mathbf{t} \rightarrow \mathbf{0}} \frac{\left|P_{n}\left(\mathbf{t}+\mathbf{r}_{1}\right)\right|^{2}}{C^{2 n}\|\mathbf{t}\|^{2 n}} \leq \lim _{t_{a} \rightarrow 0} \frac{1-g_{n}\left(t_{a}\right)}{C^{2 n}\left|t_{a}\right|^{2 n}} \\
& =\frac{1}{C^{2 n}} \lim _{t_{a} \rightarrow 0} \frac{c_{n} \int_{0}^{t_{a}}(\sin 2 \pi \xi)^{2 n+1} d \xi}{\left|t_{a}\right|^{2 n}}=0 .
\end{aligned}
$$


3.2. Tight framelets with two generators. Let us construct tight wavelet frames with two generators and vanishing moments. We obtain these tight framelets using the Unitary Extension Principle and the refinable functions introduced in the previous subsection. We adapt the constructions by Chui and He [6] and Petukhov [34] (see also [10]), which were done for dyadic dilations. Our argument is based on the Oblique Extension Principle, a method based on the Unitary Extension Principle; it was developed by Chui, He and Stöckler [7], and independently by Daubechies, Han, Ron, and Shen [13], who gave the method its name. The Oblique Extension Principle may be formulated as follows:

Theorem B. Let $A \in \mathbf{E}_{d}(\mathbb{Z})$ and let $\phi \in L^{2}\left(\mathbb{R}^{d}\right)$ be compactly supported and refinable, i.e.

$$
\widehat{\phi}\left(A^{T} \mathbf{t}\right)=P(\mathbf{t}) \widehat{\phi}(\mathbf{t}),
$$

where $P(\mathbf{t})$ is a trigonometric polynomial. Assume moreover that $|\widehat{\phi}(\mathbf{0})|=1$. Let $S(\mathbf{t})$ be another trigonometric polynomial such that $S(\mathbf{t}) \geq 0$ and $S(\mathbf{0})=1$. Assume there are trigonometric polynomials or rational functions $Q_{\ell}, \ell=1, \cdots, N$, that satisfy the OEP condition

$$
\begin{array}{rl}
S\left(A^{*} \mathbf{t}\right) P & P(\mathbf{t}) \overline{P(\mathbf{t}+\mathbf{j})}+\sum_{\ell=1}^{N} Q_{\ell}(\mathbf{t}) \overline{Q_{\ell}(\mathbf{t}+\mathbf{j})} \\
\quad= & \left\{\begin{array}{ccc}
S(\mathbf{t}) & \text { if } \quad \mathbf{j} \in \mathbb{Z}^{d}, \\
0 & \text { if } & \mathbf{j} \in\left(\left(A^{*}\right)^{-1}\left(\mathbb{Z}^{d}\right) / \mathbb{Z}^{d}\right) \backslash \mathbb{Z}^{d}
\end{array}\right.
\end{array}
$$

If

$$
\widehat{\psi_{\ell}}\left(A^{*} \mathbf{t}\right):=Q_{\ell}(\mathbf{t}) \widehat{\phi}(\mathbf{t}), \quad \ell=1, \ldots, N,
$$

then $\Psi=\left\{\psi_{1}, \ldots, \psi_{N}\right\}$ is a Parseval framelet in $L^{2}\left(\mathbb{R}^{d}\right)$ with dilation matrix $A$.

With an additional decay condition, Theorem B follows from [13, Proposition 1.11], except for the value of the frame constant, which follows from, e.g. [37, Theorem 6.5]. However, recent results of Han imply that this decay condition is redundant. Indeed, Theorem B in its present formulation is a consequence of Proposition 4, Corollary 12 and Theorem 17 in [24] (for a simpler version of Han's results in dimension one see [23]).

We need the following version of Proposition 1:

Proposition 2. Let $g_{n}$ be defined by (1), let

$$
g_{n, m}(t):=\left[g_{n}(t)\right]^{m},
$$

and let $P_{n, m}(\mathbf{t}):=\left[g_{n}\left(t_{a}\right)\right]^{m}$. Then the infinite product

$$
\prod_{j=1}^{\infty} P_{n, m}\left(\left(A^{T}\right)^{-j} \mathbf{t}\right)
$$

converges to a nonnegative continuous function $\widehat{\phi}_{n, m}$ in $L^{2}\left(\mathbb{R}^{d}\right)$ such that $\left\|\widehat{\phi}_{n, m}\right\|_{L^{2}\left(\mathbb{R}^{d}\right)} \leq 1, \widehat{\phi}_{n, m}(\mathbf{0})=1$, and $\widehat{\phi}_{n, m}$ satisfies the refinement equation

$$
\widehat{\phi}_{n, m}\left(A^{T} \mathbf{t}\right)=P_{n, m}(\mathbf{t}) \widehat{\phi}_{n, m}(\mathbf{t}), \quad \mathbf{t} \in \mathbb{R}^{d} .
$$

Moreover, the function $\phi_{n, m} \in L^{2}\left(\mathbb{R}^{d}\right)$ whose Fourier transform is $\widehat{\phi}_{n, m}$ is nonzero, compactly supported, $\left\|\phi_{n}\right\|_{L_{2}\left(\mathbb{R}^{d}\right)} \leq 1$, and the functions $\phi_{n, m}(\mathbf{t}), P_{n, m}(\mathbf{t})$, and $P_{n, m}\left(\mathbf{t}+r_{1}(A)\right)$ are even. 
8 SMOOTH COMPACTLY SUPPORTED TIGHT FRAMELETS WITH VANISHING MOMENTS

Proof. Since

$$
\left[g_{n}(0)\right]^{2 m}=1 \text { and }\left[g_{n}(t)\right]^{2 m}+\left[g_{n}\left(t+\frac{1}{2}\right)\right]^{2 m} \leq 1,
$$

the first part of the proof is established proceeding as in the proof of [3, Theorem 1] (see also [22, Lemma 2.1]). That $\left|\phi_{n, m}(\mathbf{t})\right|, P_{n, m}(\mathbf{t})$, and $P_{n, m}\left(\mathbf{t}+r_{1}(A)\right)$ are even follows by the same arguments as in the proof of Proposition 1, bearing in mind that if a function is even, so is its inverse Fourier transform.

Finally, replicating an argument of Wojtaszczyk [50, p. 79] it is easy to see that $\phi$ is compactly supported on $\mathbb{R}^{d}$.

Using Proposition 2 we can now prove:

Theorem 2. Let $h_{n, m}$ be a trigonometric polynomial on $\mathbb{R}$ such that

$$
\left|h_{n, m}(t)\right|^{2}=1-\left[g_{n, 2 m}(t)+g_{n, 2 m}\left(t+\frac{1}{2}\right)\right]
$$

let $H_{n, m}(\mathbf{t}):=h_{n, m}\left(t_{a}\right)$, and let

$$
\Psi_{n, m}=\left\{\psi_{n, m}^{(1)}, \psi_{n, m}^{(2)}\right\}
$$

be the set of functions in $L^{2}\left(\mathbb{R}^{d}\right)$ defined by

$$
\begin{aligned}
& \widehat{\psi}_{n, m}^{(1)}\left(A^{T} \mathbf{t}\right):=e^{2 \pi i t_{a}} P_{n, m}\left(\mathbf{t}+\mathbf{r}_{1}(A)\right) \widehat{\phi}_{n, m}(\mathbf{t}), \\
& \widehat{\psi}_{n, m}^{(2)}\left(A^{T} \mathbf{t}\right):=P_{n, m}(\mathbf{t}) H_{n, m}\left(A^{T} \mathbf{t}\right) \widehat{\phi}_{n, m}(\mathbf{t}) .
\end{aligned}
$$

Then $\Psi_{n, m}$ is a Parseval framelet in $L^{2}\left(\mathbb{R}^{d}\right)$ with dilation matrix $A$ and vanishing moments of order $n$, the functions $\psi_{n, m}^{(\ell)}$ have compact support, the functions $\left|\widehat{\psi}_{n, m}^{(\ell)}(\mathbf{t})\right|$ are even, and the generating function $\phi_{n, m}(\mathbf{t})$ is even and has compact support.

Proof. Let $\mathbf{r}_{1}:=\mathbf{r}_{1}(a), S(\mathbf{t}):=g_{n, 2 m}\left(t_{a}\right)+g_{n, 2 m}\left(t_{a}+\frac{1}{2}\right), Q_{1}(\mathbf{t}):=e^{2 \pi i t_{a}} P_{n, m}(\mathbf{t}+$ $\left.\mathbf{r}_{1}\right)$, and $Q_{2}(\mathbf{t}):=P_{n, m}(\mathbf{t}) H_{n, m}\left(A^{T} \mathbf{t}\right)$. Then

$$
\begin{aligned}
& S\left(A^{T} \mathbf{t}\right)\left|P_{n, m}(\mathbf{t})\right|^{2}+\sum_{\ell=1}^{2}\left|Q_{\ell}(\mathbf{t})\right|^{2}= \\
& S\left(A^{T} \mathbf{t}\right)\left|P_{n, m}(\mathbf{t})\right|^{2}+\left|P_{n, m}\left(\mathbf{t}+\mathbf{r}_{1}\right)\right|^{2}+\left|P_{n, m}(\mathbf{t}) H_{n, m}\left(A^{T} \mathbf{t}\right)\right|^{2}= \\
& \quad S\left(A^{T} \mathbf{t}\right) g_{n, 2 m}\left(t_{a}\right)+g_{n, 2 m}\left(t_{a}+\frac{1}{2}\right)+g_{n, 2 m}\left(t_{a}\right)\left(1-S\left(A^{T} \mathbf{t}\right)\right)=S(\mathbf{t}) .
\end{aligned}
$$

Moreover, bearing in mind that $A^{T} \mathbf{r}_{1} \in \mathbb{Z}^{d}$, we have

$$
\begin{aligned}
& S\left(A^{T} \mathbf{t}\right) P_{n, m}(\mathbf{t}) \overline{P_{n, m}\left(\mathbf{t}+\mathbf{r}_{1}\right)}+\sum_{\ell=1}^{2} Q_{\ell}(\mathbf{t}) \overline{Q_{\ell}\left(\mathbf{t}+\mathbf{r}_{1}\right)}= \\
& \quad S\left(A^{T} \mathbf{t}\right) P_{n, m}(\mathbf{t}) P_{n, m}\left(\mathbf{t}+\mathbf{r}_{1}\right)-P_{n, m}\left(\mathbf{t}+\mathbf{r}_{1}\right) P_{n, m}(\mathbf{t}) \\
& +P_{n, m}(\mathbf{t}) H_{n, m}\left(A^{T} \mathbf{t}\right) P_{n, m}\left(\mathbf{t}+\mathbf{r}_{1}\right) \overline{H_{n, m}\left(A^{T}\left(\mathbf{t}+\mathbf{r}_{1}\right)\right)}= \\
& S\left(A^{T} \mathbf{t}\right) g_{n, m}\left(t_{a}\right) g_{n, m}\left(t_{a}+\frac{1}{2}\right)-g_{n, m}\left(t_{a}+\frac{1}{2}\right) g_{n, m}\left(t_{a}\right)+ \\
& g_{n}\left(t_{a}\right) g_{n, m}\left(t_{a}+\frac{1}{2}\right)\left(1-S\left(A^{T} \mathbf{t}\right)\right)=0,
\end{aligned}
$$


and applying the Oblique Extension Principle we conclude that $\Psi_{n, m}$ is a Parseval framelet.

We now show that the functions in $\Psi_{n, m}$ have vanishing moments of order $n$. Since the absolute values of the eigenvalues of $A^{T}$ are greater than one, there exists $C>0$ such that $\left\|A^{T} \mathbf{t}\right\| \geq C\|\mathbf{t}\|$. Moreover, using the inequalities $0 \leq\left|\widehat{\phi}_{n, m}(\mathbf{t})\right| \leq 1$ and $g_{n, 2 m}(t)+g_{n, 2 m}\left(t+\frac{1}{2}\right) \leq 1$, we have:

$$
\lim _{\mathbf{t} \rightarrow \mathbf{0}} \frac{\left|\widehat{\psi}_{n, m}^{(1)}\left(A^{T} \mathbf{t}\right)\right|^{2}}{\left\|A^{T} \mathbf{t}\right\|^{2 n}} \leq \lim _{\mathbf{t} \rightarrow \mathbf{0}} \frac{\left|P_{n, m}\left(\mathbf{t}+\mathbf{r}_{1}\right)\right|^{2}}{C^{2 n}\|\mathbf{t}\|^{2 n}} \leq \lim _{\mathbf{t} \rightarrow \mathbf{0}} \frac{1-g_{n, 2 m}\left(t_{a}\right)}{C^{2 n}\left|t_{a}\right|^{2 n}}
$$

We can write $g_{n, 2 m}(t)=1+p_{n}(t)$ where $p_{n}$ is a trigonometric polynomial on $\mathbb{R}$ such that

$$
\lim _{t \rightarrow 0} \frac{\left|p_{n}(t)\right|}{|t|^{2 n}}=0 .
$$

Hence $\widehat{\psi}_{n, m}^{(1)}$ has a zero of order $n$ at the origin.

Now,

$$
\lim _{\mathbf{t} \rightarrow \mathbf{0}} \frac{\left|\widehat{\psi}_{n, m}^{(2)}(\mathbf{t})\right|^{2}}{\|\mathbf{t}\|^{2 n}} \leq \lim _{\mathbf{t} \rightarrow \mathbf{0}} \frac{\left|H_{n, m}(\mathbf{t})\right|^{2}}{C^{2 n}\|\mathbf{t}\|^{2 n}} \leq \lim _{\mathbf{t} \rightarrow \mathbf{0}} \frac{1-g_{n, 2 m}\left(t_{a}\right)}{C^{2 n}\left|t_{a}\right|^{2 n}}=0
$$

and we conclude that $\widehat{\psi}_{n, m}^{(2)}$ has a zero of order $n$ at the origin.

The remaining assertions follow as in the proof of Theorem 1.

\section{Smooth COMPACtLy SUPPORTED TIGHT FRAMELETS WITH VANISHING MOMENTS}

In this section we construct tight framelets that have the additional property of regularity. Whereas the tight frames in the previous section have only one or two generators, the framelets we construct here have $2^{d}+d$ generators, which, nevertheless, is a number that does not depend on the smoothness or the number of vanishing moments.

We have

Lemma 2. Let the trigonometric polynomial $g_{n, m}(t)$ be defined by (10), and

$$
P_{n, m}(\mathbf{t}):=\prod_{s=1}^{d} g_{n, m}\left(t_{s}\right) .
$$

Then $P_{n, m}(\mathbf{t})$ is nonnegative and even. Moreover,

$$
\left[P_{n, m}(\mathbf{t})\right]^{2}+\left[P_{n, m}\left(\mathbf{t}+\mathbf{r}_{1}(A)\right)\right]^{2} \leq 1 .
$$

Proof. Since $g_{n}$ is nonnegative and even, it follows that $P_{n, m}(\mathbf{t})$ is nonnegative and even.

Since $0 \leq g_{n}(t) \leq 1$, we have

$$
\left[P_{n, m}(\mathbf{t})\right]^{2}+\left[P_{n, m}\left(\mathbf{t}+\mathbf{r}_{1}(A)\right)\right]^{2} \leq g_{n}\left(t_{a}\right)+g_{n}\left(t_{a}+1 / 2\right)=1 .
$$

We also have the following analog of Proposition 1. The proof may be done using Lemma 2 and the same arguments as in the proof of Proposition 2. 
Proposition 3. The infinite product

$$
\prod_{j=1}^{\infty} P_{n, m}\left(\left(A^{T}\right)^{-j} \mathbf{t}\right)
$$

converges to a nonnegative even continuous function $\widehat{\phi}_{n, m}$ in $L^{2}\left(\mathbb{R}^{d}\right)$ such that $\left\|\widehat{\phi}_{n, m}\right\|_{L^{2}\left(\mathbb{R}^{d}\right)} \leq 1, \widehat{\phi}_{n, m}(\mathbf{0})=1$, and $\widehat{\phi}_{n, m}$ satisfies the refinement equation

$$
\widehat{\phi}_{n, m}\left(A^{T} \mathbf{t}\right)=P_{n, m}(\mathbf{t}) \widehat{\phi}_{n, m}(\mathbf{t}), \quad \mathbf{t} \in \mathbb{R}^{d} .
$$

Proof. The proof follows from Lemma 2, proceeding as in the proof of [3, Theorem 1] (see also [22, Lemma 2.1]).

The following well known result may be found in, e.g., [50, Appendix A.2]. The proof is straightforward and will be omitted.

Theorem C. Let $C^{0}$ be the class of continuous functions in $L^{2}\left(\mathbb{R}^{d}\right)$, and let $C^{r}$, $r=1,2, \ldots$ be the class of functions $f$ such that all partial derivatives of $f$ of order not greater than $r$ are continuous and in $L^{2}\left(\mathbb{R}^{d}\right)$. If

$$
|\widehat{f}(\mathbf{t})| \leq C(1+|\mathbf{t}|)^{-N-\varepsilon}
$$

for some integer $N \geq d$ and $\varepsilon>0$, then $f$ is in $C^{N-d}$.

Implicit in the proofs of [48, Lemma 3.1], [50, Proposition 5.23] and [19, Result 2.6] is the following

Theorem D. Let $A \in \mathbf{E}_{d}(\mathbb{Z})$, let $\boldsymbol{\Gamma}=\left\{\mathbf{r}_{\ell}\right\}_{\ell=0}^{|\operatorname{det} A|-1}$ be a collection of representatives of the quotient set $\left(A^{T}\right)^{-1} \mathbb{Z}^{d} / \mathbb{Z}^{d}$ such that $\mathbf{r}_{0}=\mathbf{0}$, and let $P$ be a trigonometric polynomial on $\mathbb{R}^{d}$ such that $P\left(\mathbf{r}_{\ell}\right)=0$ if $\ell \neq 0$ and $P(\mathbf{t})=1$ if and only if $\mathbf{t} \in \mathbb{Z}^{d}$. Then there exist numbers $\varepsilon, C>0$ such that

$$
\prod_{j=1}^{\infty} P\left(\left(A^{T}\right)^{-j}\right)(\mathbf{t}) \leq C|\mathbf{t}|^{-\varepsilon}, \quad \mathbf{t} \in \mathbb{R}^{d}, \quad \mathbf{t} \neq \mathbf{0} .
$$

We can now prove

Proposition 4. Let $n \in \mathbb{N}$. Then there exist two positive constants $\varepsilon$ and $C$ such that

$$
\prod_{j=1}^{\infty} P_{n, 1}\left(\left(A^{T}\right)^{-j} \mathbf{t}\right) \leq C|\mathbf{t}|^{-\varepsilon}, \quad \mathbf{t} \in \mathbb{R}^{d}, \quad \mathbf{t} \neq \mathbf{0} .
$$

Proof. From Lemma A we know that $g(t)=1$ if and only if $t \in \mathbb{Z}$, which implies that $P_{n, 1}(\mathbf{t})=1$ if and only if $\mathbf{t} \in \mathbb{Z}^{d}$. Moreover, from the same Lemma A we also know that $g(t)=0$ if and only if $t \in \mathbb{Z}+1 / 2$. This in turn implies that $P_{n, 1}\left(\mathbf{r}_{1}\right)=0$ for any full collection of representatives $\boldsymbol{\Gamma}=\left\{\mathbf{r}_{0}=(0, \ldots, 0)^{T}, \mathbf{r}_{1}=\left(r_{1}^{(1)}, \ldots, r_{1}^{(d)}\right)^{T}\right\}$ of the cosets of $\left(A^{T}\right)^{-1} \mathbb{Z}^{d} / \mathbb{Z}^{d}$ such that $\mathbf{r}_{1} \in\{0,1 / 2\}^{d}$. The assertion now follows from Theorem D.

We will now obtain some refinable functions that we will use to construct tight wavelet frames. 
Proposition 5. Let $\widehat{\phi}_{n, m}$ be the function defined as in Proposition 3. Then the function $\phi_{n, m} \in L^{2}\left(\mathbb{R}^{d}\right)$ whose Fourier transform is $\widehat{\phi}_{n, m}$ is even, nonzero, and compactly supported, and $\left\|\phi_{n, m}\right\|_{L_{2}\left(\mathbb{R}^{d}\right)} \leq 1$. Moreover, if $\varepsilon m-d>\alpha>1$, where $\varepsilon$ is such that (13) is satisfied, then $\phi_{n, m}$ is in continuity class $C^{\alpha}$.

Proof. Since $\widehat{\phi}_{n, m}$ is in $L^{2}\left(\mathbb{R}^{d}\right)$ and is nonzero, it follows that also $\phi_{n, m}$ is in $L^{2}\left(\mathbb{R}^{d}\right)$ and is nonzero. Moreover, $\left\|\phi_{n, m}\right\|_{L^{2}\left(\mathbb{R}^{d}\right)}=\left\|\widehat{\phi}_{n, m}\right\|_{L^{2}\left(\mathbb{R}^{d}\right)} \leq 1$.

Replicating the already mentioned argument of Wojtaszczyk [50, p. 79], it is easy to see that $\phi_{n, m}$ is compactly supported on $\mathbb{R}^{d}$.

It remains to prove the estimate for the degree of smoothness of $\phi_{n, m}$. By Proposition 4 we have

$$
\left|\widehat{\phi}_{n, m}(\mathbf{t})\right|=\prod_{j=1}^{\infty} P_{n, m}\left(\left(A^{T}\right)^{-j} \mathbf{t}\right)=\left(\prod_{j=1}^{\infty} P_{n, 1}\left(\left(A^{T}\right)^{-j} \mathbf{t}\right)\right)^{m} \leq C^{m}|\mathbf{t}|^{-\varepsilon m} .
$$

Therefore, since $\widehat{\phi}_{n, m}$ is continuous with $\widehat{\phi}_{n, m}(\mathbf{0})=1$,

$$
\left|\widehat{\phi}_{n, m}(\mathbf{t})\right| \leq K(1+|\mathbf{t}|)^{-\varepsilon m}
$$

Hence, if $\varepsilon m-d>\alpha>1$, Theorem $\mathrm{C}$ implies that $\phi_{n, m}$ is in continuity class $C^{\alpha}$.

We will use the following slight generalization of Theorem 3.4 of Lai and Stöckler [32]. The proof is similar, and is outlined in [41, Theorem 1], where the theorem is stated in a trivially equivalent form. We have included the constructive algorithm implicit in the original formulation of the theorem.

Theorem $\mathbf{E}$. Let $A \in \mathbb{R}^{d \times d}$ be a dilation matrix preserving the lattice $\mathbb{Z}^{d}$, let $d_{A}:=|\operatorname{det} A|$, let $\left\{\mathbf{q}_{s}\right\}_{s=0}^{d_{A}-1}$ be a full collection of representatives of the cosets of $\mathbb{Z}^{d} / A \mathbb{Z}^{d}$ with $\mathbf{q}_{0}=\mathbf{0}$, and let $\left\{\mathbf{r}_{s}\right\}_{s=0}^{d_{A}-1}$ be a full collection of representatives of the cosets of $\left(A^{T}\right)^{-1} \mathbb{Z}^{d} / \mathbb{Z}^{d}$ with $\mathbf{r}_{0}=\mathbf{0}$. Let $P(\mathbf{t})$ be a trigonometric polynomial defined on $\mathbb{R}^{d}$ that satisfies the condition

$$
\sum_{s=0}^{d_{A}-1}\left|P\left(\mathbf{t}+\mathbf{r}_{s}\right)\right|^{2} \leq 1
$$

let

and let

$$
\mathcal{P}(\mathbf{t}):=\left(P\left(\mathbf{t}+\mathbf{r}_{s}\right) ; s=0, \ldots, d_{A}-1\right)^{T},
$$

be the polyphase matrix, where $s$ denotes the row index and $l$ denotes the column index.

Let the $d_{A} \times 1$ matrix function $G(\mathbf{t})$ be defined by

$$
G(\mathbf{t}):=\mathcal{M}^{*}(\mathbf{t}) \mathcal{P}(\mathbf{t})=\left(L_{k}\left(A^{T} \mathbf{t}\right) ; k=0, \ldots, d_{A}-1\right)^{T} .
$$

Suppose that there exist trigonometric polynomials $\widetilde{P}_{1}\left(A^{T} \mathbf{t}\right), \ldots, \widetilde{P}_{M}\left(A^{T} \mathbf{t}\right)$ such that

$$
\sum_{k=0}^{d_{A}-1}\left|L_{k}(\mathbf{t})\right|^{2}+\sum_{j=1}^{M}\left|\widetilde{P}_{j}(\mathbf{t})\right|^{2}=1
$$


Let $N:=d_{A}+M$ and let the $N \times 1$ matrix function $\mathcal{G}(\mathbf{t})$ be defined by

$$
\mathcal{G}(\mathbf{t}):=\left(L_{k}\left(A^{T} \mathbf{t}\right) ; k=0, \ldots, d_{A}-1, \widetilde{P}_{j}\left(A^{T} \mathbf{t}\right) ; 1 \leq j \leq M\right)^{T},
$$

and

$$
\widetilde{\mathcal{Q}}(\mathbf{t}):=I_{N \times N}-\mathcal{G}(\mathbf{t}) \mathcal{G}^{*}(\mathbf{t}) .
$$

Let $H(\mathbf{t})$ denote the first $d_{A} \times N$ block matrix of $\widetilde{\mathcal{Q}(\mathbf{t})}$,

$$
\mathcal{Q}(\mathbf{t}):=\mathcal{M}(\mathbf{t}) H(\mathbf{t})
$$

and let $\left[Q_{1}(\mathbf{t}), \ldots, Q_{N}(\mathbf{t})\right]$ denote the first row of $\mathcal{Q}(\mathbf{t})$. Then the trigonometric polynomials $P$ and $Q_{\ell}, \ell=1, \ldots, N$, satisfy the identity

$$
\begin{aligned}
& P(\mathbf{t}) \overline{P(\mathbf{t}+\mathbf{j})}+\sum_{\ell=1}^{N} Q_{\ell}(\mathbf{t}) \overline{Q_{\ell}(\mathbf{t}+\mathbf{j})} \\
& = \begin{cases}1 & \text { if } \mathbf{j} \in \mathbb{Z}^{d}, \\
0 & \text { if } \quad \mathbf{j} \in\left(\left(A^{T}\right)^{-1}\left(\mathbb{Z}^{d}\right) / \mathbb{Z}^{d}\right) \backslash \mathbb{Z}^{d} .\end{cases}
\end{aligned}
$$

Note that (18) is a particular case of (9).

Once we have obtained the trigonometric polynomial $P_{n, m}(\mathbf{t})$ defined by (11) and an associated refinable function $\phi_{n, m} \in L^{2}\left(\mathbb{R}^{d}\right)$ defined as in Proposition 5, we may apply the algorithm described in Theorem $\mathrm{E}$ and the Oblique Extension Principle to construct a family of tight framelets $\Psi$ associated to the dilation matrix $A$. All that remains is to find trigonometric polynomials $\widetilde{P}_{j}(\mathbf{t})$ such that (17) holds.

Given $n, m \in \mathbb{N}$ and the trigonometric polynomial $g_{n, m}$ defined by (10), let $h_{n, m}$ and $u_{n, m}$ be trigonometric polynomials on $\mathbb{R}$ such that

$$
\begin{array}{r}
\left|h_{n, m}(t)\right|^{2}=1-g_{n, 2 m}(t)-g_{n, 2 m}(t+1 / 2), \quad \text { and } \\
\left|u_{n, m}(t)\right|^{2}=g_{n, 2 m}(t)+g_{n, 2 m}(t+1 / 2) .
\end{array}
$$

To see that these polynomials exist note that, for example,

$$
1-g_{n, 2 m}(t)-g_{n, 2 m}(t+1 / 2) \geq 0,
$$

and, as before, the assertion follows applying the lemma of Riesz.

We prove:

Lemma 3. Let $\Omega:=\{0,1 / 2\}^{d} \backslash \boldsymbol{\Gamma}_{A^{T}}$, let $u_{n, m}(t)$ and $h_{n, m}(t)$ be trigonometric polynomials that satisfy (19), let $P_{n, m}(\mathbf{t})$ be defined by (11), let $K=2^{d}-2$, and let $\rho: \Omega \rightarrow\{d+1, \cdots, K+d\}$ be a bijection. If

$$
\begin{aligned}
& \widetilde{P}_{n, m}^{(j)}\left(A^{T} \mathbf{t}\right):=h_{n, m}\left(t_{j}\right) \prod_{s=j+1}^{d} u_{n, m}\left(t_{s}\right), j=1, \cdots, d-1, \\
& \widetilde{P}_{n, m}^{(d)}\left(A^{T} \mathbf{t}\right):=h_{n, m}\left(t_{d}\right)
\end{aligned}
$$

and

then

$$
\widetilde{P}_{n, m}^{(\rho(\mathbf{r}))}\left(A^{T} \mathbf{t}\right):=P_{n, m}(\mathbf{t}+\mathbf{r}), \quad \mathbf{r} \in \Omega,
$$

$$
\sum_{\mathbf{r} \in \boldsymbol{\Gamma}_{A^{T}}} \mid P_{n, m}\left(\mathbf{t}+\left.\mathbf{r}\right|^{2}+\sum_{j=1}^{K+d}\left|\widetilde{P}_{n, m}^{(j)}\left(A^{T} \mathbf{t}\right)\right|^{2}=1\right.
$$


Proof. Let $\boldsymbol{\Gamma}=\boldsymbol{\Gamma}_{A^{T}}$. We have:

$$
\begin{aligned}
\sum_{\mathbf{r} \in \boldsymbol{\Gamma}}\left|P_{n, m}(\mathbf{t}+\mathbf{r})\right|^{2}+\sum_{j=1}^{K+d}\left|\widetilde{P}_{n, m}^{(j)}\left(A^{T} \mathbf{t}\right)\right|^{2} & \\
= & \sum_{\mathbf{u} \in\{0,1 / 2\}^{d}}\left|P_{n, m}(\mathbf{t}+\mathbf{u})\right|^{2}+\sum_{j=1}^{d}\left|\widetilde{P}_{n, m}^{(j)}\left(A^{T} \mathbf{t}\right)\right|^{2} \\
= & \prod_{\ell=1}^{d}\left(\sum_{s=0}^{1} g_{n, 2 m}\left(t_{\ell}+\frac{s}{2}\right)\right)+\sum_{j=1}^{d}\left|\widetilde{P}_{n, m}^{(j)}\left(A^{T} \mathbf{t}\right)\right|^{2} .
\end{aligned}
$$

Therefore

$$
\begin{aligned}
& \sum_{\mathbf{r} \in \boldsymbol{\Gamma}}\left|P_{n, m}(\mathbf{t}+\mathbf{r})\right|^{2}+\sum_{j=1}^{K+d}\left|\widetilde{P}_{n, m}^{(j)}\left(A^{T} \mathbf{t}\right)\right|^{2} \\
& =\prod_{j=1}^{d}\left|u_{n, m}\left(t_{j}\right)\right|^{2}+\sum_{j=1}^{d-1}\left(\left|h_{n, m}\left(t_{j}\right)\right|^{2} \prod_{s=j+1}^{d}\left|u_{n, m}\left(t_{s}\right)\right|^{2}\right)+\left|h_{n, m}\left(t_{d}\right)\right|^{2} \\
& =\left(\left|u_{n, m}\left(t_{1}\right)\right|^{2}+\left|h_{n, m}\left(t_{1}\right)\right|^{2}\right) \prod_{j=2}^{d}\left|u_{n, m}\left(t_{j}\right)\right|^{2} \\
& \quad+\sum_{j=2}^{d-1}\left(\left|h_{n, m}\left(t_{j}\right)\right|^{2} \prod_{s=j+1}^{d}\left|u_{n, m}\left(t_{s}\right)\right|^{2}\right)+\left|h_{n, m}\left(t_{d}\right)\right|^{2} \\
& =\prod_{j=2}^{d}\left|u_{n, m}\left(t_{j}\right)\right|^{2}+\sum_{j=2}^{d-1}\left(\left|h_{n, m}\left(t_{j}\right)\right|^{2} \prod_{s=j+1}^{d}\left|u_{n, m}\left(t_{s}\right)\right|^{2}\right)+\left|h_{n, m}\left(t_{d}\right)\right|^{2} .
\end{aligned}
$$

Repeating this procedure a finite number of times, we finally obtain

$$
\sum_{\mathbf{r} \in \boldsymbol{\Gamma}}\left|P_{n, m}(\mathbf{t}+\mathbf{r})\right|^{2}+\sum_{j=1}^{K+d}\left|\widetilde{P}_{n, m}^{(j)}\left(A^{T} \mathbf{t}\right)\right|^{2}=\left|u_{n, m}\left(t_{d}\right)\right|^{2}+\left|h_{n, m}\left(t_{d}\right)\right|^{2}=1,
$$

as we wanted to prove.

We are now ready to state the main result of this section.

Theorem 3. Let $P_{n, m}(\mathbf{t})$ be defined by (11),

$$
\begin{gathered}
L_{0}\left(A^{T} \mathbf{t}\right):=\frac{1}{\sqrt{2}}\left(P_{n, m}(\mathbf{t})+P_{n, m}\left(\mathbf{t}+\mathbf{r}_{1}(A)\right),\right. \\
L_{1}\left(A^{T} \mathbf{t}\right):=\frac{e^{i 2 \pi \mathbf{t} \cdot \mathbf{q}_{1}}}{\sqrt{2}}\left(P_{n, m}(\mathbf{t})-P_{n, m}\left(\mathbf{t}+r_{1}(A)\right),\right.
\end{gathered}
$$

and let the functions $Q_{n, m}^{(\ell)}(\mathbf{t}), \ell=1,2, \ldots, 2^{d}+d$ be the functions $Q_{\ell}(\mathbf{t}), \ell=$ $1, \ldots, N$, described in Theorem $E$ with $N=2^{d}+d$ and $P(\mathbf{t})=P_{n, m}(\mathbf{t})$. Let

$$
\widehat{\psi}_{n, m}^{(\ell)}\left(A^{T} \mathbf{t}\right):=Q_{n, m}^{(\ell)}(\mathbf{t}) \widehat{\phi}_{n, m}(\mathbf{t}), \ell=1, \ldots, 2^{d}+d,
$$

Then $\Psi_{n, m}:=\left\{\psi_{n, m}^{(\ell)}(\mathbf{x}) ; \ell=1, \ldots, 2^{d}+d\right\}$ is a Parseval framelet in $L^{2}\left(\mathbb{R}^{d}\right)$ with dilation matrix $A$, the functions $\psi_{n, m}^{(\ell)}$ are compactly supported, and have vanishing moments of order $n$. If $\varepsilon m-d>\alpha>1$ and $\varepsilon$ satisfies (13), the functions $\psi_{n, m}^{(\ell)}$, 
$\ell=1, \ldots, 2^{d}+d$, are in continuity class $C^{\alpha}$. Moreover, the generating function $\phi_{n, m}(\mathbf{t})$ is even and has compact support.

Proof. Since

$$
\left|L_{0}\left(A^{T} \mathbf{t}\right)\right|^{2}+\left|L_{1}\left(A^{T} \mathbf{t}\right)\right|^{2}=\left|P_{n, m}(\mathbf{t}+\mathbf{0})\right|^{2}+\left|P_{n, m}\left(\mathbf{t}+\mathbf{r}_{1}(A)\right)\right|^{2},
$$

Lemma 3 implies that $(20)$ is satisfied, and from Theorem E we conclude that $P_{n, m}(\mathbf{t})$ and $Q_{1}(\mathbf{t}), \ldots, Q_{\left(2^{d}+d\right)}(\mathbf{t})$ satisfy (18). Applying the Oblique Extension Principle (Theorem B), we conclude that $\Psi_{n, m}$ is a Parseval framelet.

Since the functions $Q_{n, m}^{(\ell)}(\mathbf{t})$ are trigonometric polynomials and therefore bounded on $\mathbb{R}^{d}$, the smoothness of the functions $\psi_{n, m}^{(\ell)}$ follows from (14) and Theorem C.

Since $\phi_{n, m}$ has compact support and the functions $Q_{\ell}$ are trigonometric polynomials, it follows that the functions in $\psi_{n, m}^{(\ell)}, \ell=1, \cdots, 2^{d}+d$, are compactly supported.

That $\phi_{n, m}(\mathbf{t})$ is even follows from Proposition 3.

It remains to prove that the functions in $\Psi_{n, m}$ have vanishing moments of order $n$. Since the absolute value of the eigenvalues of $A^{T}$ is greater than one, there exists $C>0$ such that $\left\|A^{T} \mathbf{t}\right\| \geq C\|\mathbf{t}\|$. Moreover, using that $0 \leq\left|\widehat{\phi}_{n, m}(\mathbf{t})\right| \leq 1$ and that $P_{n, m}(\mathbf{t})$ and $Q_{1}(\mathbf{t}), \ldots, Q_{\left(2^{d}+d\right)}(\mathbf{t})$ satisfy (18), we have:

$$
\lim _{\mathbf{t} \rightarrow \mathbf{0}} \frac{\left|\widehat{\psi}_{n, m}^{(\ell)}\left(A^{T} \mathbf{t}\right)\right|^{2}}{\left\|A^{T} \mathbf{t}\right\|^{2 n}} \leq \lim _{\mathbf{t} \rightarrow \mathbf{0}} \frac{1-\left|P_{n, m}(\mathbf{t})\right|^{2}}{C^{2 n}\|\mathbf{t}\|^{2 n}}=\lim _{\mathbf{t} \rightarrow \mathbf{0}} \frac{1-\prod_{s=1}^{d} g_{n, 2 m}\left(t_{s}\right)}{C^{2 n}\|\mathbf{t}\|^{2 n}} .
$$

Moreover, $g_{n, 2 m}(t)=1+p_{n}(t)$ where $p_{n}$ is a trigonometric polynomial on $\mathbb{R}$ such that

$$
\lim _{t \rightarrow 0} \frac{\left|p_{n}(t)\right|}{|t|^{2 n}}=0 .
$$

Therefore, combining (21) and (22), and bearing in mind that $0 \leq g_{n}(t) \leq 1$, we obtain

$$
\lim _{\mathbf{t} \rightarrow \mathbf{0}} \frac{\left|\widehat{\psi}_{n, m}^{(\ell)}(\mathbf{t})\right|^{2}}{\|\mathbf{t}\|^{2 n}} \leq \frac{1}{C^{2 n}} \lim _{\mathbf{t} \rightarrow \mathbf{0}} \sum_{s=1}^{d} \frac{\left|p_{n}\left(t_{s}\right)\right|}{t_{s}^{2 n}}=0,
$$

and we conclude that $\widehat{\psi}_{n, m}^{(\ell)}$ has a zero of order $n$ at the origin.

\section{A Bivariate COnstruction of tight Framelets}

In the previous section we used Lemma 3 to construct a set of $2^{d}+d$ generators. For $d=2$ this would yield six generators. In this section we show that if $A \in$ $\mathbf{E}_{2}(\mathbb{Z})$ we may bypass Lemma 3 and obtain generating sets with only two or three generators. This suggests that Theorem 3 could be improved considerably.

5.1. Tight wavelet frames with three generators. Two matrices $A$ and $B$ with integral entries are integrally similar if there exists a matrix $U$ with integral entries such that $|\operatorname{det} U|=1$ and $A=U^{-1} B U$. Let

$$
\begin{aligned}
A_{1}:=\left(\begin{array}{ll}
0 & 2 \\
1 & 0
\end{array}\right), A_{2}:=\left(\begin{array}{rr}
0 & 2 \\
-1 & 0
\end{array}\right), A_{3}:=\left(\begin{array}{rr}
0 & 2 \\
-1 & 1
\end{array}\right), \\
A_{4}:=\left(\begin{array}{ll}
0 & -2 \\
1 & -1
\end{array}\right), A_{5}:=\left(\begin{array}{rr}
1 & 1 \\
-1 & 1
\end{array}\right), A_{6}:=\left(\begin{array}{rr}
-1 & -1 \\
1 & -1
\end{array}\right) .
\end{aligned}
$$


The following complete classification of all matrices in $\mathbf{E}_{2}(\mathbb{Z})$ with $|\operatorname{det} A|=2$ was found by Lagarias and Wang [31, Lemma 5.2 ].

Lemma F. Let $A \in \mathbf{E}_{2}(\mathbb{Z})$. If $\operatorname{det} A=-2$ then $A$ is integrally similar to $A_{1}$, and if $\operatorname{det} A=2$ then $A$ is integrally similar to one of the matrices $A_{k}, k=2, \ldots, 6$.

We now focus on the dilation matrices $A_{k}$. Let

$$
\begin{gathered}
\Lambda:=\left\{\mathbf{q}_{0}=(0,0)^{T}, \mathbf{q}_{1}=(1,0)^{T}\right\}, \\
\Gamma_{k}:=\left\{\mathbf{r}_{0}=(0,0)^{T}, \mathbf{r}_{1}=(1 / 2,0)^{T}\right\}, \quad k=1,2,3,4, \\
\Gamma_{k}:=\left\{\mathbf{r}_{0}=(0,0)^{T}, \mathbf{r}_{1}=(1 / 2,1 / 2)^{T}\right\}, \quad k=5,6 .
\end{gathered}
$$

It is easy to see that, for $k=1, \ldots, 6, \Lambda$ is a full collection of representatives of the cosets of $\mathbb{Z}^{2} / A_{k} \mathbb{Z}^{2}, \Gamma_{k}$ is a full collection of representatives of the cosets of $\left(A_{k}^{T}\right)^{-1} \mathbb{Z}^{2} / \mathbb{Z}^{2}$.

Given $m, n \in \mathbb{N}, \mathbf{t}=\left(t_{1}, t_{2}\right)^{T} \in \mathbb{R}^{2}$ and the trigonometric polynomial $g_{n}(t)$ defined in (1), let the trigonometric polynomial $P_{n, m}(\mathbf{t})$ be defined on $\mathbb{R}^{2}$ by

$$
P_{n, m}(\mathbf{t}):=g_{n, m}\left(t_{1}\right)=\left(g_{n}\left(t_{1}\right)\right)^{m} .
$$

For convenience we have used the same notation for these polynomials and for those defined in (11). Both sets of polynomials have similar properties. For example, if $A_{k}$ is one of the dilation matrices defined in (23) and $\boldsymbol{\Gamma}_{k}=\left\{\mathbf{r}_{0}, \mathbf{r}_{1}\right\}$ is the full collection of representatives of the cosets of $\left(A_{k}^{T}\right)^{-1} \mathbb{Z}^{2} / \mathbb{Z}^{2}$ defined in (25), then

$$
\left|P_{n, m}(\mathbf{t})\right|^{2}+\left|P_{n, m}\left(\mathbf{t}+\mathbf{r}_{1}\right)\right|^{2} \leq g_{n}\left(t_{1}\right)+g_{n}\left(t_{1}+1 / 2\right)=1,
$$

and the equality only holds for an at most countable set of points. Moreover, using the same arguments as in the proofs of Proposition 1 and Proposition 2, we have the following similar result:

Proposition 6. Let $A_{k}$ be one of the matrices defined in (23), and let $P_{n, m}(\mathbf{t})$ be defined as in (26). Then the infinite product

$$
\prod_{j=1}^{\infty} P_{n, m}\left(\left(A_{k}^{T}\right)^{-j} \mathbf{t}\right)
$$

converges to an even nonnegative continuous function $\widehat{\phi}_{n, m}$ in $L^{2}\left(\mathbb{R}^{2}\right)$ such that

$$
\left\|\widehat{\phi}_{n, m}\right\|_{L^{2}\left(\mathbb{R}^{2}\right)} \leq 1
$$

$\widehat{\phi}_{n, m}(\mathbf{0})=1$ and satisfies the refinement equation

$$
\widehat{\phi}_{n, m}\left(A_{k}^{T} \mathbf{t}\right)=P_{n, m}(\mathbf{t}) \widehat{\phi}_{n, m}(\mathbf{t}), \quad \mathbf{t} \in \mathbb{R}^{2} .
$$

We now need to establish the validity of an analog of Proposition 4. However, from Lemma A, (26) and (27), we see that $P_{n, m}(\mathbf{t})$ might equal 1 without $\mathbf{t}$ being in $\mathbb{Z}^{2}$, and therefore we cannot use Theorem D directly. Nevertheless, we can prove:

Proposition 7. There exist two positive constants $\varepsilon$ and $C$ such that

$$
\prod_{j=1}^{\infty} P_{n, 1}\left(\left(A_{k}^{T}\right)^{-j} \mathbf{t}\right) \leq C|\mathbf{t}|^{-\varepsilon}, \quad \mathbf{t} \in \mathbb{R}^{2}, \quad \mathbf{t} \neq \mathbf{0} .
$$


Proof. We will prove the assertion for $A_{1}$. The proof for the other cases is similar and will be omitted. We have

$$
\prod_{j=1}^{\infty} P_{n, 1}\left(\left(A_{1}^{T}\right)^{-j} \mathbf{t}\right)=\prod_{j=1}^{\infty} H_{n, 1}\left(\left(M_{1}^{T}\right)^{-j} \mathbf{t}\right)
$$

where

$$
M_{1}:=A_{1}^{2} \quad \text { and } \quad H_{n, 1}(\mathbf{t})=H_{n, 1}\left(t_{1}, t_{2}\right):=P_{n, 1}(\mathbf{t}) P_{n, 1}\left(A_{1}^{T} \mathbf{t}\right)=g_{n}\left(t_{1}\right) g_{n}\left(t_{2}\right)
$$

We see that $H_{n, 1}(\mathbf{t})=1$ if and only if $\mathbf{t} \in \mathbb{Z}^{2}$. On the other hand, if $\Gamma_{1}=\left\{\mathbf{0}, \mathbf{r}_{1}\right\}$ is the full collection of representatives of the cosets of $\left(A_{1}^{T}\right)^{-1} \mathbb{Z}^{2} / \mathbb{Z}^{2}$ defined in (25), then $\left\{\mathbf{0}, \mathbf{r}_{1},\left(A_{1}^{T}\right)^{-1} \mathbf{r}_{1}, \mathbf{r}_{1}+\left(A_{1}^{T}\right)^{-1} \mathbf{r}_{1}\right\}$ is a full collection of representatives of the cosets of $\left(M_{1}^{T}\right)^{-1} \mathbb{Z}^{2} / \mathbb{Z}^{2}$. Furthermore, Lemma A and (27) imply that $P_{n, 1}\left(\mathbf{r}_{1}\right)=0$. Thus

$$
\begin{aligned}
H_{n, 1}\left(\mathbf{r}_{1}\right) & =P_{n, 1}\left(\mathbf{r}_{1}\right) P_{n, 1}\left(A_{1}^{T} \mathbf{r}_{1}\right)=0, \quad \text { and } \\
H_{n, 1}\left(\left(A_{1}^{T}\right)^{-1} \mathbf{r}_{1}\right) & =P_{n, 1}\left(\left(A_{1}^{T}\right)^{-1} \mathbf{r}_{1}\right) P_{n, 1}\left(\mathbf{r}_{1}\right)=0 .
\end{aligned}
$$

Moreover, since $A_{1}^{T} \mathbf{r}_{1} \in \mathbb{Z}^{2}$,

$$
\begin{aligned}
& H_{n, 1}\left(\mathbf{r}_{1}+\left(A_{1}^{T}\right)^{-1} \mathbf{r}_{1}\right)=P_{n, 1}\left(\mathbf{r}_{1}+\left(A_{1}^{T}\right)^{-1} \mathbf{r}_{1}\right) P_{n, 1}\left(A_{1}^{T} \mathbf{r}_{1}+\mathbf{r}_{1}\right) \\
& =P_{n, 1}\left(\mathbf{r}_{1}+\left(A_{1}^{T}\right)^{-1} \mathbf{r}_{1}\right) P_{n, 1}\left(\mathbf{r}_{1}\right)=0,
\end{aligned}
$$

and the assertion follows from Theorem D.

We need the following proposition. The proof is similar to that of Proposition 5 and will be omitted.

Proposition 8. Let $\widehat{\phi}_{n, m}$ be the function whose existence is established in Proposition 6. Then the function $\phi_{n, m} \in L^{2}\left(\mathbb{R}^{2}\right)$ whose Fourier transform is $\widehat{\phi}_{n, m}$ is even, nonzero, compactly supported, and $\left\|\phi_{n, m}\right\|_{L_{2}\left(\mathbb{R}^{d}\right)} \leq 1$. Moreover, if $\varepsilon m-2>\alpha>1$ where $\varepsilon$ is such that (28) is satisfied, then $\phi_{n, m}$ is in continuity class $C^{\alpha}$.

Let $h_{n, m}$ be trigonometric polynomials on $\mathbb{R}$ such that $\left|h_{n, m}(t)\right|^{2}=1-\left[g_{n}(t)\right]^{2 m}-$ $\left[g_{n}\left(t+\frac{1}{2}\right)\right]^{2 m}$, where $g_{n}$ is defined by (1).

The following theorem describes the construction of smooth tight framelets $\Psi=\left\{\psi_{0}, \psi_{1}, \psi_{2}\right\}$ in $L^{2}\left(\mathbb{R}^{2}\right)$ with dilation matrix $A_{k}$, having compact support, arbitrary degrees of smoothness. any number of vanishing moments, and symmetric generating function.

Theorem 4. Let $A_{k}$ be one of the matrices defined in (23), and let

$$
\begin{gathered}
Q_{n, m}^{(1)}(\mathbf{t}):=\frac{1}{\sqrt{2}}\left[1-g_{n, 2 m}\left(t_{1}\right)-g_{n, m}\left(t_{1}\right) g_{n, m}\left(t_{1}+1 / 2\right)\right], \\
Q_{n, m}^{(2)}(\mathbf{t}):=\frac{e^{i 2 \pi t_{1}}}{\sqrt{2}}\left[1-g_{n, 2 m}\left(t_{1}\right)+g_{n, m}\left(t_{1}\right) g_{n, m}\left(t_{1}+1 / 2\right)\right], \\
Q_{n, m}^{(3)}(\mathbf{t}):=-\widetilde{P}_{1}\left(A_{k}^{T} \mathbf{t}\right) g_{n, m}\left(t_{1}\right), \\
\widehat{\phi}_{n, m}(\mathbf{t}):=\prod_{j=1}^{\infty} P_{n, m}\left(\left(A_{k}^{T}\right)^{-j} \mathbf{t}\right), \\
\widehat{\psi}_{n, m}^{(\ell)}\left(A_{k}^{T} \mathbf{t}\right):=Q_{n, m}^{(\ell)}(\mathbf{t}) \widehat{\phi}_{n, m}(\mathbf{t}), \quad \ell=1,2,3 .
\end{gathered}
$$


Then $\Psi_{n, m}=\left\{\psi_{n, m}^{(\ell)}(\mathbf{t}), \ell=1,2,3\right\}$ is a Parseval framelet in $L^{2}\left(\mathbb{R}^{2}\right)$ with dilation matrix $A_{k}$ and vanishing moments of order $n$. Moreover, if $\varepsilon m-2>\alpha>1$, where $\varepsilon$ is such that (28) is satisfied, then $\psi_{n, m}^{(\ell)}$ is in continuity class $C^{\alpha}$ and the generating function $\phi_{n, m}(\mathbf{t})$ is even and has compact support.

Proof. Let

$$
\begin{gathered}
L_{0}(\mathbf{t}):=\frac{1}{\sqrt{2}}\left(g_{n, m}\left(t_{1}\right)+g_{n, m}\left(t_{1}+1 / 2\right)\right), \\
L_{1}(\mathbf{t}):=\frac{e^{-i 2 \pi t_{1}}}{\sqrt{2}}\left(g_{n, m}\left(t_{1}\right)-g_{n, m}\left(t_{1}+1 / 2\right)\right),
\end{gathered}
$$

and let $\widetilde{P}_{1}(\mathbf{t})$ be the trigonometric polynomial $h_{n, m}(\mathbf{t})$ that satisfies (19). Since

$$
\begin{aligned}
& \left|L_{0}(\mathbf{t})\right|^{2}+\left|L_{1}(\mathbf{t})\right|^{2}+\left|\widetilde{P}_{1}(\mathbf{t})\right|^{2} \\
= & \frac{1}{2}\left(g_{n, m}\left(t_{1}\right)+g_{n, m}\left(t_{1}+\frac{1}{2}\right)\right)^{2} \\
& +\frac{1}{2}\left(g_{n, m}\left(t_{1}\right)-g_{n, m}\left(t_{1}+\frac{1}{2}\right)\right)^{2} \\
& \quad+1-g_{n, 2 m}\left(t_{1}\right)-g_{n, 2 m}\left(t_{1}+1 / 2\right)=1,
\end{aligned}
$$

we see that (17) is satisfied. Applying the algorithm described in Theorem E we see that $P_{n, m}, Q_{n, m}^{(1)}, Q_{n, m}^{(2)}$ and $Q_{n, m}^{(3)}$ satisfy (18) for $N=3$, with $P=P_{n, m}$ and $Q_{n, m}^{(\ell)}=Q_{\ell}, \ell=1,2,3$, and from the Oblique Extension Principle we conclude that $\Psi_{n, m}$ is a Parseval framelet.

The remaining assertions follow as in the proof of Theorem 3 .

Finally, from Theorem 4 and Lemma $\mathrm{F}$ we have the following construction with three generators valid for any $A \in \mathbf{E}_{2}(\mathbb{Z})$ with $|\operatorname{det} A|=2$ :

Theorem 5. With the notation of Theorem 4, let $A \in \mathbf{E}_{2}(\mathbb{Z})$ with $|\operatorname{det} A|=2$, and let $k \in\{1, \ldots, 6\}$ be such that $A$ is integrally similar to $A_{k}$. Let $U \in \mathbb{Z}^{2 \times 2}$ be such that $A=U^{-1} A_{k} U$ and $|\operatorname{det}(U)|=1$. If

$$
\theta_{n, m}^{(\ell)}(\mathbf{t}):=\psi_{n, m}^{(\ell)}(U \mathbf{t}), \quad \ell=1,2,3,
$$

then $\Theta_{n, m}=\left\{\theta_{n, m}^{(1)}, \theta_{n, m}^{(2)}, \theta_{n, m}^{(3)}\right\} \subset L^{2}\left(\mathbb{R}^{2}\right)$ is a Parseval framelet in $L^{2}\left(\mathbb{R}^{2}\right)$ with dilation matrix $A$, and the functions $\theta_{n, m}^{(\ell)}$ have compact support and vanishing moments of order $n$. Moreover, if $\varepsilon m-2>\alpha>1$, where $\varepsilon$ is such that (28) is satisfied, then the functions $\theta_{n, m}^{(\ell)}$ are in continuity class $C^{\alpha}$.

Proof. Since $U^{-1} \mathbb{Z}^{2}=\mathbb{Z}^{2}$, the assertion that $\Theta$ is a Parseval framelet readily follows by a change of variable of the form $t \rightarrow U^{-1} t$.

Moreover, (29) implies that $\widehat{\psi}_{n, m}^{(\ell)}$ has a zero of order $n$ at the origin if and only if $\widehat{\theta}_{n, m}^{(\ell)}$ has a zero of order $n$ at the origin.

Let $\alpha$ be an integer such that $\varepsilon m-2>\alpha>1$, where $\varepsilon$ is such that (28) is satisfied. Since $\psi_{n, m}^{(\ell)}$ is in continuity class $C^{\alpha}$, applying the chain rule we conclude that also $\theta_{n, m}^{(\ell)}$ is in continuity class $C^{\alpha}$.

The reason why Theorems 4 and 5 have been stated separately is that, whereas we have a constructive procedure to obtain the wavelets in Theorem 4, Theorem 5 does not provide a constructive method, for it relies on an existence theorem. 
However, bearing in mind that the eigenvalues of $A_{2}, A_{3}, A_{4}, A_{5}$ and $A_{6}$ are $\pm \sqrt{2} i$, $(1 \pm \sqrt{7} i) / 2,(-1 \pm \sqrt{7} i) / 2,1 \pm i$ and $-1 \pm i$ respectively, and in view of Lemma $\mathrm{F}$, it is not difficult to determine which of these matrices is integrally similar to a given $A \in \mathbf{E}_{2}(\mathbb{Z})$ with $|\operatorname{det} A|=2$. The same observation applies to the relationship between Theorem 6 and Theorem 7 below.

5.2. Tight wavelet frames with two generators. Using the results we obtained in Subsection 3.2, we have the following:

Theorem 6. Let $A_{k}$ be one of the matrices defined in (23), and let $\mathbf{t}=\left(t_{1}, t_{2}\right)^{T} \in$ $\mathbb{R}^{2}$. Let $P_{n, m}(\mathbf{t})$ be defined in (26), and let

$$
\widehat{\phi}_{n, m}(\mathbf{t}):=\prod_{j=1}^{\infty} P_{n m}\left(\left(A_{k}^{T}\right)^{-j} \mathbf{t}\right)
$$

Let $h_{n, m}$ be a trigonometric polynomial on $\mathbb{R}$ that satisfies (19), and let $H_{n, m} \in$ $L^{2}\left(\mathbb{R}^{2}\right)$ be defined by $H_{n, m}(\mathbf{t}):=h_{n, m}\left(t_{1}\right)$. Let

$$
\Psi_{n, m}=\left\{\psi_{n, m}^{(1)}, \psi_{n, m}^{(2)}\right\}
$$

be the set of functions defined by

$$
\begin{aligned}
& \widehat{\psi}_{n, m}^{(1)}\left(A_{k}^{T} \mathbf{t}\right):=e^{2 \pi i t_{1}} \overline{P_{n, m}\left(\mathbf{t}+\mathbf{r}_{1}\right)} \widehat{\phi}_{n, m}(\mathbf{t}), \\
& \widehat{\psi}_{n, m}^{(2)}\left(A_{k}^{T} \mathbf{t}\right):=P_{n, m}(\mathbf{t}) H_{n, m}\left(A_{k} \mathbf{t}\right) \widehat{\phi}_{n, m}(\mathbf{t}) .
\end{aligned}
$$

Then $\Psi_{n, m}$ is a Parseval framelet with dilation matrix $A_{k}$. The functions $\psi_{n, m}^{(\ell)}$ are square-integrable on $\mathbb{R}^{2}$ and have compact support. In addition, $\Psi_{n, m}$ has vanishing moments of order $n$. If $\varepsilon m-2>\alpha>1$, where $\varepsilon$ is such that (28) is satisfied, then $\psi_{n, m}^{(\ell)}$ is in continuity class $C^{\alpha}$. Moreover, the generating function $\phi_{n, m}(\mathbf{t})$ is even and has compact support.

That $\Psi_{n, m}=\left\{\psi_{n, m}^{(1)}, \psi_{n, m}^{(2)}\right\}$ is a Parseval framelet can be proved as in the proof of Theorem 2. The remaining assertions follow as in the proof of Theorem 4. The details will be omitted.

The main result in this subsection is

Theorem 7. Let $A \in \mathbf{E}_{2}(\mathbb{Z})$ with $|\operatorname{det} A|=2$ and let $k \in\{1, \ldots, 6\}$ be such that $A$ is integrally similar to $A_{k}$. Let $U \in \mathbb{Z}^{2 \times 2}$ with $|\operatorname{det}(U)|=1$ be such that $A=U^{-1} A_{k} U$. Let $m, n \in \mathbb{N}$ and $\mathbf{t}=\left(t_{1}, t_{2}\right)^{T} \in \mathbb{R}^{2}$, let $P_{n, m}(\mathbf{t})$ be defined in (26), let the function $\widehat{\phi}_{n, m} \in L^{2}\left(\mathbb{R}^{d}\right)$ be defined as in Proposition 6, and let $\Psi_{n, m}=\left\{\psi_{n, m}^{(1)}, \psi_{n, m}^{(2)}\right\}$ be the set of functions defined in Theorem 6. If

$$
\theta_{n, m}^{(\ell)}(\mathbf{t}):=\psi_{n, m}^{(\ell)}(U \mathbf{t}) \quad \ell=1,2,
$$

then $\Theta_{n, m}=\left\{\theta_{n, m}^{(1)}, \theta_{n, m}^{(2)}\right\} \subset L^{2}\left(\mathbb{R}^{2}\right)$ is a tight framelet with dilation matrix $A$, and the functions $\theta_{n, m}^{(\ell)}$ have compact support. In addition, $\Theta_{n, m}$ has vanishing moments of order $n$. Moreover, if $\varepsilon m-2>\alpha>1$ where $\varepsilon$ is such that (28) is satisfied, then the functions $\theta_{n, m}^{(\ell)}$ are in continuity class $C^{\alpha}$.

Theorem 7 can be proved in a way similar to that of Theorem 5, using Theorem 6 instead of Theorem 4 . 


\section{REFERENCES}

[1] A. Ayache, Some methods for constructing nonseparable, orthonormal, compactly supported wavelet bases, Appl. Comput. Harmon. Anal. 10 (2001) 99-111.

[2] E. Belogay and Y. Wang, Arbitrarily smooth orthogonal nonseparable wavelets in $\mathbb{R}^{2}$, SIAM J. Math. Anal. 30 (1999), no. 3, 678-697.

[3] M. Bownik, Tight frames of multidimensional wavelets, J. Fourier Anal. Appl. 3, (1997) no. $5,525-542$.

[4] A. S. Cavaretta, W. Dahmen and C.A. Micchelli, Stationary subdivision. Mem. Amer. Math. Soc. 93 (1991), no. 453.

[5] Charles K. Chui; An Introduction to Wavelets, Academic Press, Inc. 1992.

[6] C.K. Chui and W. He, Compactly supported tight frames associated with refinable functions. Appl. Comput. Harmon. Anal. 8 (2000), no. 3, 293-319.

[7] C.K. Chui, W. He and J. Stöckler, Compactly supported tight and sibling frames with maximum vanishing moments, Appl. Comput. Harmonic Anal. 13 (2002) 224-262.

[8] C.K. Chui, W. He, J. Stöckler and Q. Sun, Compactly supported tight affine frames with integer dilations and maximum vanishing moments. Frames. Adv. Comput. Math. 18 (2003), no. 2-4, 159-187.

[9] C. K. Chui and J. Lian, A Construction of compactly supported symmetric and antisymmetric orthonormal wavelets with scale =3. Appl. Comput. Harmon. Anal. 2 (1995), no. 1, 21-51.

[10] Ole Christensen; An Introduction to frames and Riesz bases, Birkhäser, Boston, 2003.

[11] I. Daubechies, Orthonormal bases of compactly supported wavelets, Comm. Pure Appl. Math. 41 (1988) 906-996.

[12] I. Daubechies, Ten lectures on wavelets, SIAM, Philadelphia, 1992.

[13] I. Daubechies, B. Han, A. Ron and Z. W. Shen, Framelets: MRA-based constructions of wavelet frames, Appl. Comput. Harmon. Anal. 14 (2003), 1-46.

[14] B. Dong and Z. Shen, Pseudo-splines, wavelets and framelets. Appl. Comput. Harmon. Anal. 22 (2007), no. 1, 78-104.

[15] M. Ehler, On multivariate compactly supported bi-frames, J. Fourier Anal. Appl. 13 (2007), no. $5,511-532$.

[16] M. Ehler and B. Han, Wavelet bi-frames with few generators from multivariate refinable functions, Appl. Comput. Harmon. Anal. 25 (2008), no. 3, 407-414.

[17] T.N.T. Goodman, C.A. Micchelli, G. Rodriguez, S. Seatzu, Spectral factorization of Laurent polynomials, Adv. Comput. Math. 7 (1997), 429-454.

[18] K. Gröchening and W. R. Madych, Multiresolution analysis, Haar bases and self-similar tillings of $R^{n}$, IEEE Trans. Inform. Theory, 38 (1992), no. 2, 556-568.

[19] K. Gröchenig and A. Ron, Tight compactly supported wavelet frames of arbitrarily high smoothness, Proc. Amer. Math. Soc. 126 (1998) 1101-1107.

[20] B. Han, On dual wavelet tight frames, Applied Comput. Harmon. Anal. 4 (1997) 380-413.

[21] B. Han, Symmetric orthonormal scaling functions and wavelets with dilation factor 4, Adv. Comput. Math. 8 (1998), no. 3, 221-247.

[22] B. Han, Compactly supported tight wavelet frames and orthonormal wavelets of exponential decay with a general dilation matrix. Approximation theory, wavelets and numerical analysis, (Chattanooga, TN, 2001). J. Comput. Appl. Math. 155 (2003), no. 1, 43-67.

[23] B. Han, Pairs of frequency-based nonhomogeneous dual wavelet frames in the distribution space, Appl. Comput. Harmon. Anal. 29 (2010), no. 3, 330-353.

[24] B. Han, Nonhomogeneous wavelet systems in high dimensions, App. Comput. Harmon. Anal. 32 (2012), no. 2, 169-196.

[25] B. Han, Matrix splitting with symmetry and symmetric tight framelet filter banks with two high-pass filters. Appl. Comput. Harmon. Anal. 35 (2013), no. 2, 200-227.

[26] B. Han, Symmetric tight framelet filter banks with three high-pass filters. Appl. Comput. Harmon. Anal. 37 (2014), no. 1, 140-161.

[27] B. Han and Q. Mo, Symmetric MRA tight wavelet frames with three generators and high vanishing moments, Appl. Comput. Harmon. Anal. 18 (2005), no. 1, 67-93.

[28] E. Hernández and G. Weiss; A first course on Wavelets, CRC Press Inc., 1996.

[29] E. Kovačević and M. Vetterli, Nonseparable multidimensional perfect reconstruction filter banks and wavelet bases for $\mathbb{R}^{n}$, IEEE Trans. Inform. Theory 38 (1992), no.2, 533-555. 
[30] A. V. Krivoshein, On construction of multivariate symmetric MRA-based wavelets, Appl. Comput. Harmon. Anal. 36 (2014), no. 2, 215-238.

[31] J. C. Lagarias and Y. Wang, Haar type orthonormal wavelet bases in $R^{2}$. (English) J. Fourier Anal. Appl. 2, No.1, 1-14 (1995).

[32] M.-J. Lai and J. Stöckler, Construction of multivariate compactly supported tight wavelet frames, Appl. Comput. Harmon. Anal. 21 (2006) 324-348.

[33] Y. Meyer, Ondelettes et opérateurs. I, Hermann, Paris, 1996 [English Translation: Wavelets and operators, Cambridge University Press, 1992]

[34] A. Petukhov, Explicit construction of framelets, Appl. Comput. Harmon. Anal. 11 (2001), 313-327.

[35] A. Petukhov, Framelets with many vanishing moments, Approx. Theory X (St. Lous, MO, 2001) 425-432, Innov. Apl. Math., Vanderbitt Univ. Press, Nashville, N, 2002.

[36] A. Petukhov, Construction of symmetric orthogonal bases of wavelets and tight wavelet frames with integer dilation factor, Appl. Comput. Harmon. Anal. 17 (2004), 198-210.

[37] A. Ron and Z.W. Shen, Affine systems in $L^{2}\left(\mathbb{R}^{d}\right)$ : The analysis of the analysis operator, J. Funct. Anal. 148 (1997) 408-447.

[38] A. Ron and Z.W. Shen, Compactly supported tight affine frames in $L^{2}\left(\mathbb{R}^{d}\right)$, Math. Comp. 67 (1998) 191-207.

[39] A. Ron and Z.W. Shen, Construction of compactly supported afine frames in $L^{2}\left(\mathbb{R}^{d}\right)$, in: K.S. Lau (Ed.), Advances in Wavelets, Springer-Verlag, New York, 1998, pp. 27-49.

[40] M. Salvatori and P. M. Soardi, Multivariate tight affine frames with a small number of generators, J. Approx. Theory 127 (2004), no. 1, 61-73.

[41] A. San Antolín and R. A. Zalik, Some smooth compactly supported tight framelets, Comm. Math. and Appl. No 3 vol 3 (2012), 345-353.

[42] A. San Antolín and R. A. Zalik, Some Bivariate Smooth Compactly Supported Tight Framelets with Three Generators, Abstract and Applied Analysis, vol. 2013, Article ID 818907, 7 pages, (2013). DOI: 10.1155/2013/818907.

[43] A. San Antolín and R. A. Zalik, A family of nonseparable scaling functions and compactly supported tight framelets, J. Mathematical Analysis and Applications 404 (2013), 201-211. DOI: $10.1016 /$ j.jmaa.2013.02.040.

[44] I. W. Selesnick, Smooth wavelet tight frames with zero moments, Appl. Comput. Harmon. Anal. 10 (2001), 163-181.

[45] M. Skopina, On construction of multivariate wavelets with vanishing moments. Appl. Comput. Harmon. Anal. 20 (2006), no. 3, 375-390.

[46] M. Skopina, Tight wavelet frames. (Russian) Dokl. Akad. Nauk 419 (2008), no. 1, 26-29; translation in Dokl. Math. 77 (2008), no. 2, 182-185.

[47] M. Skopina, On construction of multivariate wavelet frames. Appl. Comput. Harmon. Anal. 27 (2009), no. 1, 55-72.

[48] R.S. Strichartz, Wavelets and self-affine tilings, Constructive Approximation 9, (1993) No. $2-3,327-346$.

[49] V. Velisavljević, B. Beferull-Lozano, M. Vetterli and P. L. Dragotti, Directionlets: Anisotropic Multidirectional Representation With Separable Filtering, IEEE Trans. Image Proc. (15) (2006), 1916-1933.

[50] P. Wojtaszczyk, A mathematical introduction to wavelets, London Math. Soc., Student Texts $37,1997$. SPAIN.

Departamento de Análisis Matemático, Universidad de Alicante, 03080 Alicante,

E-mail address: angel.sanantolin@ua.es

Department of Mathematics and Statistics, Auburn University, Auburn, Al. 36849 5310

E-mail address: zalik@auburn.edu 